\title{
Revista Colombiana de

\section{Capítulo 6. Selección de los candidatos para el ingreso a las clínicas de falla cardiaca}

\section{Carlos A. Arias* y Jeffrey Castellanos}

\author{
Sección de Cardiología, Clínica Colsánitas, Bogotá, Colombia
}

Recibido el 17 de noviembre de 2015; aceptado el 12 de enero de 2016 Disponible en Internet el 1 de febrero de 2016

\section{PALABRAS CLAVE Insuficiencia cardiaca; Pronóstico; Estudios de seguimiento}

\section{KEYWORDS}

Heart failure; Prognosis; Follow-up studies

\begin{abstract}
Resumen
Introducción: Las unidades de insuficiencia cardiaca son una estrategia de atención de buena calidad y con altos estándares de costo-efectividad, con impacto en la calidad de vida, aumento de la supervivencia y disminución de las rehospitalizaciones y de los costos en una patología con impacto en la salud pública.

Objetivo: Formular estrategias que ayuden a definir la población que se beneficia del seguimiento en las Clínicas de falla cardiaca.

Metodología: Revisión narrativa de la literatura disponible.

Conclusión: La población de pacientes con falla cardiaca que son de alto riesgo definidos por criterios como clase funcional II a iv persistente, 2 o más hospitalizaciones por falla cardiaca descompensada en los últimos doce meses, falla cardiaca de novo o criterios sugestivos de progresión de la enfermedad son quienes más se benefician del seguimiento en programas multidisciplinarios.

(c) 2016 Publicado por Elsevier España, S.L.U. en nombre de Sociedad Colombiana de Cardiología y Cirugía Cardiovascular. Este es un artículo Open Access bajo la licencia CC BY-NC-ND (http://creativecommons.org/licenses/by-nc-nd/4.0/).
\end{abstract}

\section{Chapter 6. Selection of candidates for admission to heart failure clinics}

\begin{abstract}
Introduction: Cardiac failure units are a good quality care strategy, have high cost-effectiveness standards and impact quality of life, increase survival and reduce rehospitalisation and the costs of a disorder which affects public health.

Objetive: To formulate strategies towards defining the population who will benefit from followup in heart failure clinics.

Methodology: Narrative review of the available literature.

Conclusion: The population of high risk heart failure patients defined by criteria such as functional class ॥l to persistent Iv, 2 or more admissions to hospital due to decompensated heart
\end{abstract}

\footnotetext{
* Autor para correspondencia.

Correo electrónico: carlosandresmd@hotmail.com (C.A. Arias).
} 
failure in the past 12 months, de novo heart failure or criteria suggestive of progression of the disease are those who benefit most from follow-up in multidisciplinary programmes.

(c) 2016 Published by Elsevier España, S.L.U. on behalf of Sociedad Colombiana de Cardiología y Cirugía Cardiovascular. This is an open access article under the CC BY-NC-ND license (http://creativecommons.org/licenses/by-nc-nd/4.0/).

\section{Introducción}

Los candidatos deben tener un diagnóstico claro de insuficiencia cardiaca, con criterios clínicos y de exámenes bien establecidos.

\section{Definición de falla cardiaca}

La falla cardíaca es un síndrome complejo, caracterizado por anomalías de la función ventricular y de la regulación neurohormonal, síntomas de intolerancia al ejercicio y retención de líquidos, además de signos de hipertensión venosa pulmonar y/o sistémica o de bajo gasto cardiaco, atribuibles a la disfunción mecánica de uno o ambos ventrículos ${ }^{1,2}$.

En este aspecto es importante el proceso de educación médica continuada del personal que labora en el hospital en lo pertinente a la adherencia a guías de detección y diagnóstico de la insuficiencia cardiaca y a la interpretación correcta de ecocardiografías, electrocardiografías, radiografías de tórax, péptidos natriuréticos y demás exámenes que permitan definir severidad, comorbilidad y compromiso multisistémico ${ }^{3,4}$. Se debe procurar reducir la variabilidad en la calidad del diagnóstico en los diferentes niveles de complejidad del personal de atención como meta fundamental desde el inicio del programa y su desempeño se debe someter a evaluación constante ${ }^{5,6}$.

La detección de la población con insuficiencia cardiaca y criterios de alto riesgo se debe realizar mediante la búsqueda activa e integrada de los casos en las diferentes dependencias del hospital, como el servicio de urgencias, hospitalización general y cuidados intensivos. Para lograr este objetivo se debe contar con el apoyo del personal médico y de enfermería que trabaja allí y es de gran utilidad la divulgación de los criterios de ingreso al programa mediante el uso de material escrito o alertas en la historia clínica electrónica de la institución ${ }^{7}$. Una vez detectados los candidatos es importante resaltar que la evaluación integral del paciente anticipa a problemas en el seguimiento; por esta razón conviene evaluar la severidad de la enfermedad cardiovascular y los factores psicosociales que pueden influir en el deterioro de la condición de salud del paciente y generar nuevos ingresos al hospital ${ }^{8,9}$.

Una vez confirmado el diagnóstico de falla cardiaca, el siguiente paso es definir si el paciente tiene criterios de estabilidad y determinar sus necesidades para definir si puede continuar el seguimiento en atención primaria o si se trata de un paciente de alto riesgo que debe ingresar a la unidad de falla cardiaca ${ }^{10-12}$. Los criterios para seleccionar los candidatos a ingresar a las clínicas de falla cardiaca son:
- Clase funcional II a Iv persistente.

- Una o más hospitalizaciones por falla cardiaca descompensada en los últimos doce meses.

- Criterios sugestivos de progresión de la enfermedad ${ }^{13}$

- Biológicos: deterioro de la función renal persistente, anemia, elevación de péptidos natriuréticos.

- Funcionales: deterioro de la clase funcional según la clasificación de la NYHA.

- Portadores de dispositivos de alto voltaje y resincronización cardiaca o candidatos a los mismos.

- Nutricionales: caquexia cardiaca, pérdida ponderal mayor a $10 \%$ en seis meses y albúmina menor de $2,5 \mathrm{mg} / \mathrm{dl}$.

- Falla cardiaca de novo

A su vez, los criterios para que el seguimiento se realice en una unidad de atención primaria con apoyo periódico de la clínica de falla cardiaca son:

- Pacientes con riesgo social: persona con dependencia, que vive sola o con familia con capacidad limitada de apoyo por edad o enfermedad.

- No aceptación del paciente y/o familia o cuidador.

- Paciente sin ingresos recientes por insuficiencia cardiaca o estable en clase funcional I-II.

- Comorbilidad significativa (otras enfermedades concomitantes que hayan condicionado un ingreso hospitalario en los últimos doce meses diferentes a falla cardiaca descompensada).

- Alteración cognitiva moderada a severa.

- Barreras que dificulten acceder al programa, por ejemplo uso de ambulancia para su traslado.

- Paciente en quien ya se ha demostrado falta de adherencia dentro del programa de insuficiencia cardiaca.

\section{Conflicto de intereses}

Los autores declaran no tener ningún conflicto de intereses.

\section{Bibliografía}

1. Mcmurray JJV, Adamopoulos S, Anker SD, Auricchio A, Böhm M, Dickstein K, et al. ESC Guidelines for the diagnosis and treatment of acute and chronic heart failure 2012. Eur J Heart Fail. 2012;14:803-69.

2. Yancy CW, Jessup M, Bozkurt B, Butler J, Casey DE, Drazner $\mathrm{MH}$, et al. 2013 ACCF/AHA guideline for the management of heart failure: a report of the American College of Cardiology Foundation/American Heart Association Task Force on Practice Guidelines. J Am Coll Cardiol. 2013;62:e147-239. 
3. Pani L, Pecorelli S, Rosano G, Anker SD, Peracino A, Fregonese $\mathrm{L}$, et al. Steps forward in regulatory pathways for acute and chronic heart failure. Eur J Heart Fail. 2015;17:3-8.

4. Buggey J, Mentz RJ, Galanos AN. End-of-life heart failure care in the United States. Heart Fail Clin. 2015;11:615-23.

5. Baudendistel I, Noest S, Peters-Klimm F, Herzberg H, Scherer $\mathrm{M}$, Blozik $\mathrm{E}$, et al. Bridging the gap between patient needs and quality indicators: a qualitative study with chronic heart failure patients. Patient Prefer Adherence. 2015;9:1397-405.

6. Freeman GK. Holding relationships in general practice: What are they? How do they work? Are they worth having? Brit J GenPract. 2011;61:487-8.

7. Comín-Colet J, Verdu J, Vallespín E, Cleries M, Bustins M, Mendoza $L$, et al. Eficacia de un programa integrado hospitalatención primaria para la insuficiencia cardiaca: análisis poblacional sobre 56.742 pacientes. Rev Esp Cardiol. 2014;67: 283-93.

8. McAlister FA, Lawson FM, Teo KK, Armstrong PW. A systematic review of randomized trials of disease management programs in heart failure. Am J Med. 2001;110:378-84.
9. McAlister FA, Stewart S, Ferrua S, McMurray JJ. Multidisciplinary strategies for the management of heart failure patients at high risk for admission: a systematic review of randomized trials. J Am Coll Cardiol. 2004;44:810-9.

10. Krumholz HM, Amatruda J, Smith GL, Mattera JA, Roumanis SA, Radford MJ, et al. Randomized trial of an education and support intervention to prevent readmission of patients with heart failure. J AM Coll Cardiol. 2002;39:83-9.

11. Phillips CO, Wright SM, Kern DE, Singa RM, Shepperd S, Rubin HR. Comprehensive discharge planning with postdischarge support for older patients with congestive heart failure: a metaanalysis. JAMA. 2004;291:1358-67.

12. Nagarajan V, Tang WH. Management of comorbid conditions in heart failure: a review. Med Clin North Am. 2012;96: 975-85.

13. Conde-Martel A, Hernández-Meneses M. Prevalence and prognostic meaning of comorbidity in heart failure. Rev Clin Esp. 2015. Oct 8. pii: S0014-2565(15)00221-0. 